\title{
HSGS Investigation for Limiting the Secondary Arc on UHV Parallel Lines
}

\author{
Zhang Xu \\ North China Electric Power University \\ Changping, Beijng ,102206 \\ zhangxuabb@163.com
}

\author{
Yan Xiaoqing,Xu Zhenyu \\ North China Electric Power University \\ Changping, Beijng , 102206 \\ e-mail:name@xyz.com
}

\begin{abstract}
The HSGS characteristic for secondary arc limitation on UHV parallel lines is investigated versus singlephase to ground faults and cross-country faults, respectively by theoretical analysis and RTDS verification. The influential factors such as electrostatic induction, electromagnetic coupling, line length, HSGS single end closing and the secondary arc resistance are involved. The results show that secondary arc current and recovery voltage of cross-country faults are higher than that of single-phase to ground faults. The secondary arc current and recovery voltage increase with the increasing line length and load level. HSGS single end closing doesn't help extinguishing the arc, while the high secondary arc resistance does. The conclusion shows that HSGS with low resistance and double-end quick closing in middle and short lines could extinguish secondary arc effectively, therefore the single-phase reclosing succeeds. Advice would be provided for the construction of UHV transmission lines in China.
\end{abstract}

Keywords- UHV, High-Speed Grounding Switch (HSGS); parallel lines, single-phase reclosing, arc current, recovery voltage

\section{INTRODUCTION}

All manuscripts must be in English. These guidelines include complete descriptions of the fonts, spacing, and related information for producing your proceedings manuscripts. Please follow them and if you have any questions, direct them to the production editor in charge of your proceedings at Conference Publishing Services (CPS): Phone +1 (714) 821-8380 or Fax +1 (714) 761-1784.

The secondary arc current and recovery voltage of 1000 $\mathrm{kV}$ UHV parallel transmission lines are high after singlephase to ground faults and cross-country faults, causing the failure of selective pole reclosing[1]. Two methods are mainly utilized to limit the secondary arc current and recovery voltage. One is to equip HSGS (High Speed Grounding Switching) and the other is to install neutral reactor in the neutral of shunt reactors.

Yutaka Goda mainly analyzed on the UHV single-circuit transmission line how to use HSGS to extinguish the secondary arc[4]. R.M.Hasibar showed the advantage of using HSGS on $500 \mathrm{kV}$ transmission line and compared HSGS cost to reactor. R.M.Hasibar and Y.Yamagata studied transient characteristics when HSGS is closed[5-7]. M.Jannati researched how long the secondary arc could be extinguished when HSGS and reactor are applied respectively[8]. Shang Liqun showed that HSGS was suitable for $500 \mathrm{kV}$ middle and short lines[9]. Han Yanhua used distributed parameters to analyze arc current on a $500 \mathrm{kV}$ transmission line with HSGS, showing how to calculate secondary arc current in different disposition and studying the impact of fault resistance on the current[10].

As the backbone of smart grid in the future, the security and reliability of UHV parallel transmission lines are of great importance. The secondary arc and recovery voltage versus cross-country faults are much severer than that versus singlephase to ground faults, significantly threatening the successful reclosing. Therefore the corresponding investigations have to be carried out versus cross-country faults, even though its possibility is low, as well as the single-phase to ground fault. This paper analyzes arc current and recovery voltage characteristics in single-phase to ground faults and cross-country faults.The HSGS characteristic on UHV parallel lines for limiting the secondary arc and corresponding influential factors, such as the line length, load level, HSGS single end closing, secondary arc resistance and other factors are mainly studied.

The corresponding simulation results show the correctness of theoretical research. Proposal is provided for the construction of UHV transmission lines in the future.

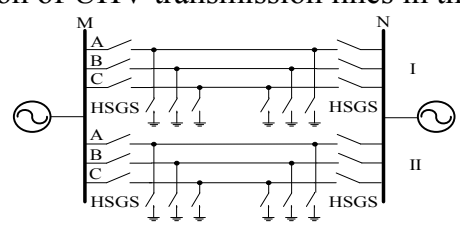

Figure 1. Structure map of UHV parallel lines with HSGS

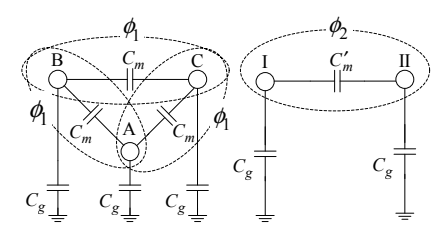

Figure 2. Capacitances and inductances of UHV parallel lines

\section{High SpeEd Grounding Switch}

Shown in Fig.1, bus $\mathrm{M}$ is connected with the supply end, bus $\mathrm{N}$ is connected with the load end. HSGS is installed in the UHV transmission lines at both ends of each line. The majority of secondary arc current loads from HSGS, 
therefore the secondary arc would be extinguished as soon as quickly.

Operating sequence of HSGS:

(1) Single-phase to ground transient fault occurs, primary arc generates;

(2) Breakers are tripped at both ends of the fault phase, the primary arc is off, the secondary arc generates;

(3) HSGS close, the secondary arc is extinguished;

(4)HSGS open;

(5)Breakers are closed, power lines resume operation

\section{THEORETICAL ANALYSIS}

When single-phase to ground faults or cross-country faults happen in UHV lines, electrostatic induction and electromagnetic coupling between fault phase and health lines in both circuits influence the secondary arc current and recovery voltage. Assuming the line is fully transported, as Fig. 2 shows, $\mathrm{Cm}$ represents the capacitance between two lines in a circuit. C'm represents the capacitance of two circuits. $\Phi 1$ represents electromagnetic coupling between two lines in a circuit. $\Phi 2$ represents electromagnetic coupling of two circuits.

As formula (1), (2) show, the secondary arc current and recovery voltage contains capacitance parts (ISC,USC) and inductance parts (ISL ,USL).

$$
\begin{aligned}
& I_{S}=I_{S C}+I_{S L} \\
& U_{S}=U_{S C}+U_{S L}
\end{aligned}
$$

\section{A. Electrostatic Induction Current and Voltage}

For example, IB to ground fault (IBg):

$I_{S C}=\left(E_{I A}+E_{I C}\right) \omega C_{m}+\left(E_{I I A}+E_{I I B}+E_{I C}\right) \omega C_{m}^{\prime}$

$$
U_{S C}=\frac{\left(E_{I A}+E_{I C}\right) C_{m}+\left(E_{I I A}+E_{I I B}+E_{I I C}\right) C_{m}^{\prime}}{C_{g}+3 C_{m}^{\prime}+2 C_{m}}
$$

For example, IBIIB to ground fault (IBgIIBg):

$I_{S C}=\left(E_{I A}+E_{I C}\right) \omega C_{m}+\left(E_{I I A}+E_{I C}\right) \omega C_{m}^{\prime}$

$$
U_{S C}=\frac{\left(E_{I A}+E_{I C}\right) C_{m}+\left(E_{I I A}+E_{I C}\right) C_{m}^{\prime}}{C_{g}+2 C_{m}^{\prime}+2 C_{m}}
$$

Formula (3), (4), (5), (6) show ISC would be higher in a longer line while USC don't change with line length significantly, ISC and USC in cross-country fault is larger than single-phase to ground fault.

\section{B. Electromagnetic Induction Current and Voltage}

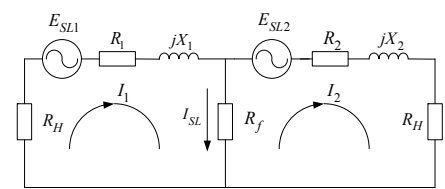

Figure 3. Electromagnetic Induction Current

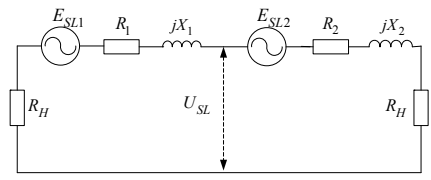

Figure 4. The influence of load on US

$$
I_{S L}=\frac{\left(E_{S L 1}-E_{S L 2}\right) R_{H}}{\left(R_{H}+R_{L}+R_{f}\right)\left(R_{H}+R_{R}+R_{f}\right)-R_{f}^{2}}
$$

In formula (7), ESL1, ESL2, are the electromagnetic induction voltage produced by health lines. RL and RR are line impedance on both ends of fault point.Lm1, Lm2, L'm1, L'm2 are the mutual inductance correspondingly. RH is HSGS resistance, $\mathrm{Rf}$ is secondary arc resistance.ISL is related with load, fault location and RH .If RH is $0 \mathrm{ohms}$, the load influence is little. If RH isn't 0 ohms, ISL would be influenced much by load. Meanwhile in the center of line, load has no influence, while it does in other parts.

$$
U_{S L}=\frac{E_{S L 1}\left(R_{R}+R_{H}\right)-E_{S L 2}\left(R_{L}+R_{H}\right)}{2 R_{H}+R_{L}+R_{R}}
$$

In the formula (8) USL is related with load, fault location and RH .And if RH is 0 ohms, loading effects can be ignored. If RH isn't, when the fault occurred in the mid-point of line, load affects little; when a fault occurs in line the other position, impact of the load increases.

\section{C. single end closing}

If only one end of HSGS close, HSGS's diversion of arc current will be reduced. To take $\mathrm{M}$ end closes, $\mathrm{N}$ end opens as an example:

$$
\begin{aligned}
& I_{S L}=\frac{E_{S L 1}}{R_{H}+R_{L}+R_{f}} \\
& U_{S L}=E_{S L 1}
\end{aligned}
$$

\section{NUMERICAL VERIFICATION}

Parameters of UHV parallel lines between Huainan and Huxi are used in the simulation. Line length is $0 \sim 500 \mathrm{~km}$ in Fig. 5 and Fig. 6.And it is $163 \mathrm{~km}$ with HSGS in Fig. $7 \sim$ Fig. 12 , which is the same as UHV lines of zhebei-Huxi $(163 \mathrm{~km})$.

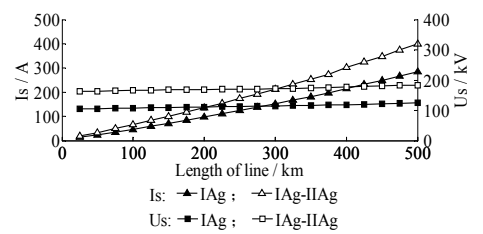

Figure 5. IS and US of UHV parallel lines

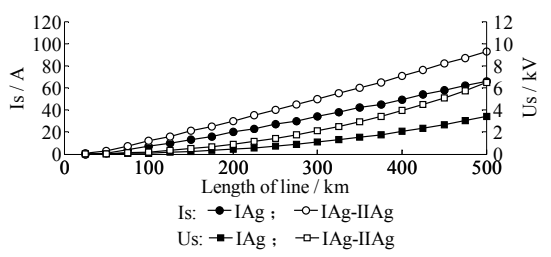

Figure 6. IS and US of UHV parallel lines with HSGS 


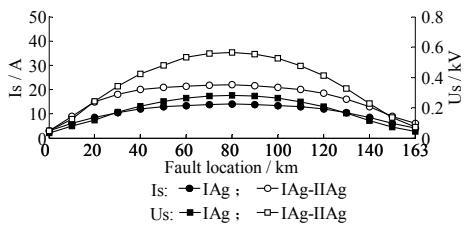

Figure 7. IS and US in IAg and IAgIIAg

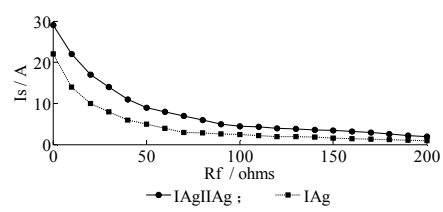

Figure 8. IS of different $\mathrm{Rf}$

Shown in Fig. 5, when IAg fault happens, IS grows with increasing of the line length. US doesn't change significantly and is approximately $110 \mathrm{kV}$. When IAgIIAg fault happens, IS is greater than IAg fault and US is about $170 \mathrm{kV}$. If no methods are taken, the secondary arc current may not be automatically extinguished. When HSGS is used in UHV parallel lines, compared with Fig. 5, IS and US are significantly reduced. However IS and US grow as line length increases. And IS and US in IAgIIAg fault are also higher than in IAg fault. Therefore cross-country faults should be considered, especially in UHV parallel lines. It is well known that resistance of $\mathrm{RH}$ is very small .which is good for lowering IS and US.As shown in Fig.7, IAg or IAgIIAg happen at different point along $163 \mathrm{~km}$ UHV parallel lines with HSGS, IS and US get the highest value at middle point, and lower as they near the ends. When Rf increases, IS of IAg or IAgIIAg decreases. IS get maximum if $\mathrm{Rf}$ close to 0ohms.

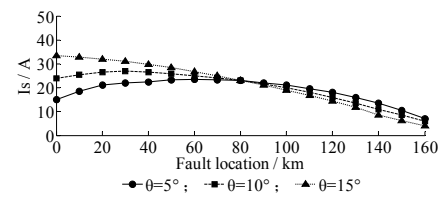

Figure 9. IS of IAgIIAg in different load

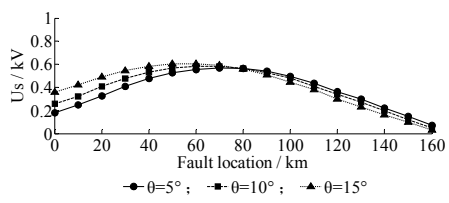

Figure 10. US of IAgIIAg in different load

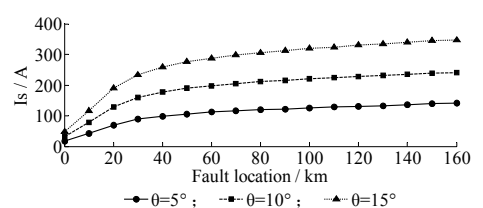

Figure 11. IS of IAgIIAg fault while only HSGS of M end is closed

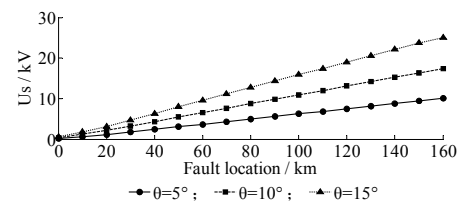

Figure 12. US of IAgIIAg fault while only HSGS of M end is closed

Power load increases as power angle $\theta$ grows up gradually. In Fig. 9 10, from $M$ end to middle point, IS and US increase as power load grows up, while they decrease from middle point to $\mathrm{N}$ end. That is because $\mathrm{RH}$ is usually small. It is worth noting that IS and US don't change at middle point whatever power load increases or not. As shown in Fig. 11 12, HSGS of M end is closed, while $\mathrm{N}$ end is open, when IAg or IAgIIAg happen. IS and US increase as fault point farther from $M$ end or power load grows up. So HSGS of single end close is disadvantage for extinguishing arc.

\section{CONCLUSION}

For UHV parallel lines with HSGS, in order to quickly extinguish the secondary arc to ensure the success of singlephase reclosing, impact of two circuits on IS and US should be considered. Middle point is the worst point. Lowering RH tends to reduce load influence. Meanwhile, it is good for decreasing IS and US. HSGS of single end closed is disadvantage. So HSGS of double end should be closed nearly at the same time. Low Rf may keep secondary arc existing for a long time. HSGS would better to be used in middle or short UHV parallel lines.

\section{REFERENCES}

[1] M.Ramold; J.Jager.Enhancement of the Reliability of Extra and Ultra High Voltage Transmission Systems [C] //2009 IEEE Power Tech Conference, June 28th 2009-July 2nd 2009, Bucharest, Romania.

[2] E D.Kimbark.Selective-Pole Switching of Long Double-Circuit EHV Line [J].IEEE Trans.on Power Apparatus and Systems, 1976, 95(1),PP : 219-229.

[3] SHANG Liqun; SHI Wei.Study of the Secondary Arc Extinction at EHV Double Circuit Transmission Lines [J].Automation of Electric Power Systems, 2005, 29(10),PP : 60-72.

[4] Yutaka Goda; Shoji Matsuda; Tsuginori Inaba; Yuzo Ozaki.Forced extinction characteristics of secondary arc on UHV (1000kV class) transmission lines [J].IEEE Transactions on Power Delivery, 1993, 8(3),PP:1322-1330.

[5] R.M.Hasibar; A.C.Legate; J.Brunke.The application of high-speed grounding switches for single-pole reclosing on $500 \mathrm{kV}$ power systems [J].IEEE Transactions on Power Apparatus and Systems, 1981, 100(4),PP : 1512-1515.

[6] R.M.Hasibar; A.C.Legate; J.Brunke.The application of high-speed grounding switches for single-pole reclosing on $500 \mathrm{kV}$ power systems [J].IEEE Transactions on Power Apparatus and Systems, 1981, 100(4),PP : 1512-1515.

[7] Y.Yamagata; K.Tanaka; Y.Terasawa.Development of interrupting chamber for $1000 \mathrm{kV}$ HSGS with a delayed-zero-current interrupting performance [C] // Proceedings of EMPD '95, 1995, 1,PP : 404-409.

[8] M.Jannati; B.Vahidi; S.H.Hosseinian.A Novel Approach for Optimizing Dead Time of Extra High Voltage Transmission Lines Optimization of Electrical and Electronic Equipment [C] // OPTIM 2008, 2008,PP : 215-220. 
[9] SHANG Liqun; SHI Wei.Application of High Speed Grounding Switches in Double-Circuit Transmission Lines [J].High Voltage Engineering, 2004, 30(8),PP : 14-16.

[10] HAN Yanhua; FAN Yue; SHI Wei.Secondary Arc Extinction by
High Speed Grounding Switches [J].JOURNAL OF XI' AN JIAOTONG UNIVERSITY, 2000, 34(8),PP : 15-17. 